\title{
CAPACIDADE ABSORTIVA EM INSTITUIÇÕES DE ENSINO SUPERIOR: UMA SISTEMATIZAÇÃO DA LITERATURA
}

\author{
ABSORPTIVE CAPACITY IN HIGHER EDUCATION \\ INSTITUTIONS: A SYSTEMATIZATION OF LITERATURE
}

\section{CAPACIDAD ABSORTIVA EN INSTITUCIONES DE ENSEÑANZA SUPERIOR: UNA SISTEMATIZACIÓN DE LA LITERATURA}

\author{
Rogério Ciotti \\ Mestre em Administração na Universidade do Oeste de \\ Santa Catarina (UNOESC) / Professor e coordenador do \\ Curso de Tecnologia em Análise e Desenvolvimento de \\ Sistemas da Faculdade Anglicana de Erechim (FAE), \\ Brasil \\ rogeriociotti@gmail.com
}

\section{Jacir Favretto}

Pós-Doutor pela o Programa de Pós-Doutorado da A Faculdade de Economia, Administração e Contabilidade da Universidade de São Paulo (FEA/USP) / Professor titular da Universidade do Oeste de Santa Catarina (UNOESC), Brasil / Professor da Universidade do Contestado (UnC), Brasil

jacirfa@gmail.com

\author{
Contextus \\ ISSNe 2178-9258 \\ Organização: Comitê Científico Interinstitucional \\ Editor Científico: Carlos Adriano Santos Gomes \\ Avaliação: double blind review pelo SEER/OJS \\ Recebido em 05/12/2016 \\ Aceito em 20/12/2017 \\ $2^{\mathrm{a}}$ versão aceita em 27/12/2017
}

\section{RESUMO}

A busca das organizações por conhecimento externo e a posterior incorporação deste no seu ambiente interno são fatores determinantes para alcançar e sustentar vantagens competitivas. A chamada capacidade absortiva consiste na capacidade de a empresa adquirir, assimilar, transformar, explorar e utilizar, para fins comerciais, o conhecimento proveniente do ambiente externo. Por meio de técnicas bibliométricas, o objetivo do presente estudo é analisar a produção acadêmica sobre capacidade absortiva no contexto das instituições de ensino superior, de modo a traçar um mapa dos gaps para novas contribuições acadêmicas na área. Foram selecionados 4231 trabalhos nas bases de dados Spell, Scielo, ScienceDirect, Ebsco, Scopus e Web of Science. Após aplicação de regras de exclusão, restaram 26 artigos para análise.

Palavras-chave: Capacidade Absortiva. Instituições de Ensino Superior. Competitividade. Bibliometria.

\begin{abstract}
The search of organizations for external knowledge and the subsequent incorporation of this in their internal environment are determining factors to achieve and sustain competitive advantages. The so-called absorptive capacity consists of the company's ability to acquire, assimilate, transform, exploit and use knowledge from the external environment for commercial purposes. By means of bibliometric techniques, the aim of this study is to analyze the academic production on absorptive capacity in the context of higher education institutions in order to map the gaps for new academic contributions in the area. 4231 papers were selected from Spell, Scielo, ScienceDirect, Ebsco, Scopus and Web of Science databases. After applying exclusion rules, 26 articles remained for analysis.
\end{abstract}

Keywords: Absorptive Capacity. Higher education institutions. Competitiveness. Bibliometrics.

RESUMEN

La búsqueda de las organizaciones por conocimiento externo y la posterior incorporación de éste en su ambiente interno son factores determinantes para alcanzar y sostener ventajas competitivas. La llamada capacidad 
absorbente consiste en la capacidad de la empresa para adquirir, asimilar, transformar, explotar y utilizar, con fines comerciales, el conocimiento proveniente del ambiente externo. Por medio de técnicas bibliométricas, el objetivo del presente estudio es analizar la producción académica sobre capacidad absorbente en el contexto de las instituciones de enseñanza superior, para trazar un mapa de los gaps para nuevas contribuciones académicas en el área. Se seleccionaron 4231 trabajos en las bases de datos Spell, Scielo, ScienceDirect, Ebsco, Scopus y Web of Science. Después de la aplicación de reglas de exclusión, quedaron 26 artículos para análisis.

Palabras clave: Capacidad Absortiva. Instituciones de Enseñanza Superior. Competitividad. Bibliometría.

\section{INTRODUÇÃO}

O grande desafio das organizações em desenvolver uma capacidade adaptativa ao dinamismo do ambiente é recorrente para alcançar e sustentar vantagens competitivas. Assim, passa a ser fundamental buscar conhecimento constantemente e encontrar respostas sobre as condições mais propícias para a contínua criação, difusão e incorporação de produtos, serviços e sistemas organizacionais.

A aquisição de conhecimento externo e sua posterior incorporação no ambiente interno são fatores determinantes para o desempenho das organizações, fenômeno denominado de capacidade absortiva - CA (NONAKA; TAKEUCHI, 1997; COHEN; LEVINTHAL， 1990; ZHARA; GEORGE, 2002). A CA explica porque algumas empresas são mais eficientes do que outras em inovação, competitividade e desempenho organizacional.

Instituições de ensino superior (IES) brasileiras, expostas ao ritmo acelerado de transformações no seu campo de atuação, percebem sua volatilidade e preocupam-se com a possibilidade de perderem competitividade. Para Balestrin (2007), a missão e funções da universidade e das IES dependem fundamentalmente do contexto social. Estudar, investigar e descobrir as complexidades e consequências do fenômeno da mercantilização da educação superior é de fundamental importância para as instituições, os sistemas e a sociedade.

Nesse contexto, estudos sobre o tema CA nas IES têm certo grau de significância, já que lhes permite se adaptarem ao ambiente externo, proporcionando se manterem competitivas. A questão é: como se encontra o panorama de estudos envolvendo CA no contexto das IES? O objetivo do presente estudo é analisar a produção acadêmica sobre capacidade absortiva no contexto das IES, de modo a traçar um mapa dos gaps para novas contribuições acadêmicas na área.

Durante a pesquisa, foram localizados apenas dois estudos com objetivo semelhante, um deles (PICOLI; TAKAHASHI, 2016) sobre instituições de ensino básico, visando compreender o 
processo recursivo de absorção e de aprendizagem organizacional. Baseou-se em mecanismos de integração social empregados pelo Programa Paraná Digital em duas instituições de ensino público de Curitiba - Paraná, no período de 2007 a 2013. O outro estudo, realizado em instituições de ensino superior, investigou o desenvolvimento da capacidade absortiva de uma universidade brasileira, diante do desafio de atuar em um projeto colaborativo junto à indústria de semicondutores (OLIVEIRA; BALESTRIN, 2015).

Para alcançar o objetivo, utilizou-se o método de pesquisa bibliométrica. A coleta de dados foi realizada utilizando-se dos termos de busca: "capacidade absortiva", "instituições de ensino superior", "absorptive capacity" e "higher education institutions". As bases de dados pesquisadas foram: Spell, Scielo, ScienceDirect, Ebsco, Scopus e Web of Science. Os resultados da busca totalizaram 4231 trabalhos. A primeira filtragem se deu através do download dos artigos disponíveis de forma gratuita, restando então 909. Em uma segunda seleção, foram excluídos os artigos analisando apenas os títulos, restando 413 trabalhos. Na terceira seleção, os critérios de exclusão foram a leitura do resumo e, se necessário, de todo o estudo, restando um total de 26 artigos para análise.
A seguir é apresentada uma revisão bibliográfica sobre o constructo capacidade absortiva, um histórico sobre IES no contexto Brasileiro para uma melhor compreensão do cenário analisado.

\section{CAPACIDADE ABSORTIVA}

A compreensão de como as empresas podem efetivamente desenvolver uma capacidade adaptativa frente ao dinamismo do ambiente é recorrente. Porque dedicam tempo e dinheiro e o resultado pode não ser o desejado devido a sua capacidade de absorção. Fenômeno definido como a capacidade da empresa em adquirir, assimilar, transformar, explorar e utilizar o conhecimento proveniente do ambiente externo (COHEN; LEVINTHAL,1990; ZHARA e GEORGE, 2002; KOZA e LEWIN, 1998).

A capacidade de absorção permite que as empresas aprendam a fazer algo completamente diferente para inovar em processos, serviços e produtos (COHEN; LEVINTHAL, 1990; VAN DEN BOSCH; VOLBERDA; DE BOER, 1999; SZULANSKI, 1996; ZAHRA; GEORGE, 2002; LANE; KOKA; PATHAK, 2006; LICHTENTHALER; LICHTENTHALER, 2009).

O constructo capacidade absortiva teve o marco inicial com a publicação de 
Cohen e Levinthal (1990), esses identificaram três dimensões do constructo: a habilidade da empresa de reconhecer o valor do novo conhecimento externo; a capacidade da empresa de assimilar o novo conhecimento, internalizando-o, e por último, a terceira que diz respeito à capacidade de comercializar o novo conhecimento. A partir daí o constructo tem despertado significativo interesse da comunidade científica e tem sido empregado para explicar uma série de fenômenos organizacionais (LANE; KOKA; PATHAK, 2002).

Para Cohen e Levinthal (1990), a empresa deve possuir três antecedentes organizacionais para desenvolver essa habilidade, que são: o conhecimento prévio e acumulado da empresa, atividades de pesquisa e desenvolvimento $(\mathrm{P} \& \mathrm{D})$ e uma boa comunicação interna.

O conhecimento prévio é descrito como conhecimento que se acumula ao longo da história da empresa, tais como habilidades dos membros individuais, utilização de métodos de resolução de problemas e linguagem compartilhada. A geração do novo conhecimento ocorre à medida que o indivíduo faz associações entre conhecimento anterior e o novo, e aplica no seu ambiente (COHEN e LEVINTHAL, 1990; ZAHRA; GEORGE, 2002).
Outro fator considerado é o nível de educação, treinamento técnico e experiência adquirida ao longo do tempo pelos empregados em determinado campo do conhecimento, quanto maior, mais aptos estarão para assimilar e transformar o novo conhecimento, gerando a necessidade de aquisição de novos conhecimentos a partir do conhecimento prévio em determinado campo. Essa situação facilita a seleção, interpretação e aplicação do novo (NUNES, 2012; COHEN E LEVINTHAL, 1990; ZAHRA; GEORGE, 2002).

$\mathrm{O}$ processo os projetos de $\mathrm{P} \& \mathrm{D}$ contribui e intensifica a acumulação de conhecimento técnico e experiências relacionadas na resolução de problemas. Considerado determinante para a acumulação de conhecimento no campo de atuação da firma. Pois desencadeia a criação de capacidade absortiva como um subproduto do investimento em P\&D (COHEN e LEVINTHAL, 1990).

Ter canais de comunicação externos que forneçam a informação relevante, assim como canais internos, que garantam sua distribuição e compartilhamento são fatores determinantes. Além disso, possuir uma linguagem e símbolos predominante no ambiente, garante que o conhecimento obtido externamente seja distribuído de forma clara e eficiente, de forma vertical e 
horizontal (COHEN e LEVINTHAL, 1990).

Em uma reconceituação do constructo Zahra e George (2002), o definiram CA como um conjunto de rotinas e processos pelos quais empresas adquirem, assimilam, transformam e exploram conhecimentos. Nessa reconceituação a CA recebe quatro dimensões e é dividida em capacidade absortiva potencial e realizada.

A capacidade absortiva potencial é o que permite a organização ser receptiva ao conhecimento externo, isto é, adquirir, analisar, interpretar e compreender este conhecimento. Já a capacidade absortiva realizada, se dá pela capacidade da empresa em transformar e explorar esse novo conhecimento. Essa distinção se dá em função do argumento que firmas podem compreender bem problemas técnicos complexos, mas podem não ser capazes de utilizar esse conhecimento para inovação e para fins comerciais (ZAHRA e GEORGE, 2002; LANE e LUBATKIN, 1998; COHEN e LEVINTHAL, 1990). Para Zahra e George (2002), as quatro dimensões devem ser analisadas como sendo complementares entre si dentro do constructo.

Aquisição refere-se à capacidade de uma empresa em identificar e adquirir conhecimento gerado externamente. A assimilação refere-se a rotinas e processos que permitem analisar, processar, interpretar e compreender o conhecimento. Transformação significa a capacidade de uma empresa para desenvolver e aperfeiçoar suas rotinas combinando conhecimento adquirido com o conhecimento existente. Exploração são rotinas que permitem as empresas aperfeiçoar, ampliar e alavancar competências existentes, exigem conhecimento e recuperação do que já foi criado e internalizado para uso (ZAHRA; GEORGE, 2002).

As empresas não podem explorar o conhecimento sem antes de adquiri-lo. Da mesma forma, podem adquirir e assimilar conhecimento, mas podem não ter a capacidade de transformar e explorar para a geração de lucro (ZAHRA e GEORGE, 2002; COHEN e LEVINTHAL, 1990). Sendo assim, as capacidades da empresa se tornam complementares dentro do constructo e devem manter um equilíbrio.

Empresas que se esforçam na aquisição e assimilação de novos conhecimentos externos, ou seja, em capacidade absortiva potencial são capazes de renovar continuamente, mas podem não ganhar benefícios a partir exploração desse conhecimento. Por outro lado, empresas que se esforçam na transformação e exploração, capacidade de absortiva realizada, podem obter lucros através da exploração, mas podem não ser capazes de 
responderem às mudanças ambientais (JANSEN, BOSCH, VOLBERDA, 2005; AHUJA e LAMPERT, 2001). Por esse motivo que um equilíbrio entre as duas é fundamental.

As rotinas existentes na organização auxiliam na complementaridade entre as dimensões do constructo, essas podem ser sistematizadas ou criadas sob demanda. Rotinas sistematizadas permitem que as empresas sustentem a exploração de conhecimentos por tempo prolongado. Já rotinas criadas sob demanda são aquelas que surgem por acaso, durante a execução de processos (ZAHRA; GEORGE, 2002). Além das dimensões e rotinas internas, fontes externas de conhecimento também foram pesquisadas e contribuíram com a literatura.

Vega-Jurado et al. (2008) definem duas fontes externas de conhecimento para as organizações: a Científica e a Industrial. Não alterando a definição inicial de Cohen e Levinthal (1990), que mencionam como fontes de conhecimento internas o próprio setor de Pesquisa e Desenvolvimento (P \& D), e de conhecimento externo, os concorrentes, bem como governo e laboratórios universitários. Vega-Jurado et al. (2008) dividem o constructo em capacidade absortiva científica e capacidade absortiva industrial.

A capacidade de absortiva científica é a capacidade da empresa de absorver conhecimentos oriundos das universidades, institutos tecnológicos e eventos científicos. Já a capacidade absortiva industrial é a capacidade da empresa de assimilar e explorar o conhecimento proveniente de seus parceiros industriais, como clientes, concorrentes, fornecedores, dentre outros (VEGA-JURADO et al.,2008; MUROVEC; PRODAN, 2009).

Outras reconceituações surgiram com a apresentação de novos frameworks e revisões da literatura, porém, não havendo inclusão de novas dimensões apenas alterando a ordem e incluindo subgrupos nessas dimensões, conforme quadro 1. 
Quadro 1 - Reconceituações do constructo

\begin{tabular}{|c|c|c|}
\hline Autores & Dimensões & Reconceituação \\
\hline $\begin{array}{ll}\text { Cohen e } & \text { Levinthal } \\
(1989,1990) & \end{array}$ & $\begin{array}{lcl}\text { Reconhecimento de } & \text { valor, } \\
\text { assimilação e aplicação } & \end{array}$ & $\begin{array}{l}\text { Conhecimento prévio da organização como } \\
\text { fator determinante }\end{array}$ \\
\hline Zahra e George (2002) & $\begin{array}{l}\text { Aquisição, assimilação, } \\
\text { transformação e exploração }\end{array}$ & $\begin{array}{l}\text { Acrescentam mais uma dimensão ao } \\
\text { constructo e dividem em Capacidade } \\
\text { Absortiva Potencial e Capacidade Absortiva } \\
\text { Realizada, além disso, argumentam que se } \\
\text { trata de uma capacidade dinâmica. }\end{array}$ \\
\hline $\begin{array}{l}\text { Jansen; Bosch; } \quad \text { Volberda } \\
(2005)\end{array}$ & $\begin{array}{l}\text { Aquisição, } \\
\text { transformação e explorimilação, }\end{array}$ & 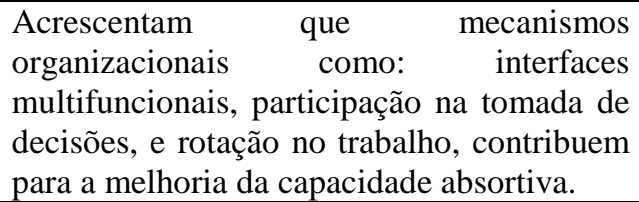 \\
\hline Todorova e Durism (2007) & $\begin{array}{ll}\text { Reconhecimento } & \text { de valor, } \\
\text { aquisição, } & \text { assimilação, } \\
\text { transformação e exploração }\end{array}$ & $\begin{array}{l}\text { Analisaram o modelo de Zhara e George } \\
(2002) \text { e reintroduziram o processo de } \\
\text { Reconhecimento de valor que havia sido } \\
\text { retirado na reconceituação. Além disso, } \\
\text { argumentam que a transformação e } \\
\text { assimilação ocorrem em um mesmo } \\
\text { momento. }\end{array}$ \\
\hline Lane, Koka e Pathak (2006) & $\begin{array}{l}\text { Reconhecimento de valor, } \\
\text { assimilação e transformação, } \\
\text { aplicação e exploração. }\end{array}$ & $\begin{array}{l}\text { Reuniram em um modelo semelhante ao } \\
\text { apresentado por Cohen e Levinthal } \\
(1989,1990) \text {, todas as reconceituações } \\
\text { anteriores, porém de uma forma diferenciada } \\
\text { (Figura 4). }\end{array}$ \\
\hline Vega-Jurado et al. (2008) & $\begin{array}{ll}\text { Reconhecimento } & \text { de valor, } \\
\text { aquisição, } & \text { assimilação, } \\
\text { transformação e exploração }\end{array}$ & $\begin{array}{l}\text { Contribuem com a literatura apresentando } \\
\text { dois conceitos de capacidade de absortiva: a } \\
\text { Científica e a Industrial. }\end{array}$ \\
\hline
\end{tabular}

Fonte: adaptada pelo autor (2016).

A identificação dos mecanismos de CA executados pelas organizações, permite diagnosticar por que algumas empresas são ineficazes sob mudanças no ambiente externo, enquanto outros prosperam sob as mesmas condições. A mera exposição ao conhecimento externo não é suficiente para garantir que a organização irá internaliza-lo com sucesso (PENNINGS e HARIANTO, 1992). A empresa precisa desenvolver capacidades combinatórias para absorver o conhecimento externo, transforma-lo e explorá-lo. Principalmente sobre mecanismos ligados a estruturas de gestão e relações sociais, interfaces multifuncionais, participação na tomada de decisão, redes de socialização, rotação no trabalho, fatores que influenciam a CA (CAMPION; CHERASKIN; STEVENS, 1994; WEICK, 1979; COHEN e LEVINTHAL, 1990; COHEN e BAC DAYAN, 1994).

Mecanismos organizacionais associados com capacidades de coordenação (interfaces multifuncionais, participação e rotação no trabalho) reforçam a capacidade de absorção potencial, enquanto mecanismos organizacionais associados a capacidades de socialização (redes de conexão e táticas de socialização) fortalecem a capacidade de 
absorção realizada (JANSEN, VAN DEN

BOSCH, VOLBERDA, 2005).

\section{INSTITUIÇÕES DE ENSINO SUPERIOR}

As primeiras instituições universitárias a nível mundial foram constituídas em Bolonha e em Paris, durante os séculos XI e XII. Sob a tutela da Igreja, possuíam total autonomia perante os poderes locais e atendiam principalmente aos filhos dos nobres (ROSSATO, 1998). Já no século XIX, o principal objetivo na universidade passou a ser não mais formar bons cristãos, como no período feudal, mas formar bons cidadãos, capazes de cumprir as funções que o estado e a sociedade passaram a exigir (TOBÍO; PÉREZ, 2005). Na segunda metade do século $\mathrm{XX}$, quase todos os países do mundo tinham universidades, e novos desafios estavam começando a surgir como, por exemplo, a demanda por acesso de outras classes sociais que não a da elite (BERTOLIN, 2007).

As IES, enfrentam mudanças para se adaptarem as exigências da sociedade e do mercado, ou seja, se adequam aos novos contextos sociais. Para Boaventura de Souza Santos (2004), nas últimas décadas, as funções relativas à dimensão cultural da educação superior têm perdido relevância para as funções demandadas pelo mercado, tais como a prestação de serviço para as indústrias e as empresas e a formação rápida profissionalizante.

As primeiras IES Brasileiras foram fundadas pelos jesuítas durante o período colonial. Logo, após a transferência da sede do poder metropolitano para o Brasil, em 1808, foram nomeadas como cátedras para formação de profissionais atuantes preferencialmente na academia militar nas áreas de Medicina e Engenharia (CUNHA, 2000).

A primeira grande multiplicação das escolas de ensino superior se deu no período que vai de 1891 até 1910, quando foram criadas no Brasil 27 escolas superiores (CUNHA, 2000). A multiplicação se deu pela grande procura por formação de nível terciário devido as transformações econômicas e institucionais. Além de, após o processo de federalização do Brasil, serem fundadas faculdades estaduais $\mathrm{e}$ privadas que se fiscalizadas pelo governo federal poderiam emitir diplomas no mesmo formato das instituições públicas.

No início dos anos 1970, houve segunda grande expansão impulsionada pela pressão de diversos segmentos da sociedade brasileira, que se tornava cada vez mais urbana e industrializada (SCHWARTZMAN, 1993). Tendo destaque ao setor privado, que foi mais 
dinâmico e cresceu mais rapidamente que o público.

Até então apenas faculdades existiam no Brasil, em 1909 surgiu a primeira Universidade Brasileira em Manaus oferecia os cursos de Engenharia, Direito, Medicina, Farmácia, Odontologia e de formação de oficiais da Guarda Nacional. Em 1911 a Universidade de São Paulo, e, em 1920 a universidade do Rio de Janeiro que resultou da união das faculdades federais de Medicina e de Engenharia (descendentes das cátedras criadas em 1808 e 1810), primeira universidade duradoura do Brasil (CUNHA, 2000).

O ensino superior teve dois grandes marcos na constituição da República Brasileira. O primeiro no ano de 1891, que lhe facultou a possibilidade de existência, o segundo foi a Constituição de 1988 que, reafirmando o princípio liberal, manteve o ensino superior livre à iniciativa privada, se respeitadas as normas gerais da educação (SAMPAIO, 2011).

A Constituição de 1988 criou um instrumento importante para o setor privado: a possibilidade de liberar-se do controle burocrático do antigo Conselho Federal de Educação (CFE), especialmente no que diz respeito à criação e extinção de cursos na sede e ao remanejamento do número de vagas oferecidas. Essa mudança ocasionou a expansão das universidades. Sendo possível não enfrentar situações em que os cursos não obtivessem demandas. Caso ocorresse por sua conta própria poderia finaliza-lo.

Entre 1985 e 1996, o número de universidades privadas mais do que triplicou (de 20 para 64), evidenciando a percepção do setor de que instituições maiores e autônomas, com uma oferta mais diversificada de cursos, teriam vantagens competitivas na disputa da clientela (CUNHA, 2000). Porém, nem todas as instituições podem desfrutar dessa autonomia devido as classificações do ensino superior brasileiro.

Os últimos anos podem ser reconhecidos por algumas alterações que ocorreram na educação superior, destacando-se, entre outros: massificação e progressiva heterogeneidade dos estudantes, redução de investimentos do setor público, novas orientações na formação e a incorporação das novas tecnologias e o próprio ensino a distância (ZABALZA, 2004). Também por meio de aquisições e fusões, um número considerável de pequenas instituições, a maioria delas faculdades isoladas espalhadas no território nacional, passou a integrar grandes grupos educacionais. 
Tabela 1 - Número de instituições de educação superior no Brasil - 2000-2013

\begin{tabular}{cc}
\hline Ano & Número de instituições \\
\hline 2000 & 1180 \\
2002 & 1637 \\
2004 & 2013 \\
2007 & 2281 \\
2010 & 2378 \\
2011 & 2365 \\
2012 & 2416 \\
2013 & 2391 \\
\hline
\end{tabular}

Fonte: INEP - Censo da Educação Superior (2004, 2007, 2013).

Nos dados apresentados na Tabela 1, há uma predominância da categoria privada, em 2013 ela representou $87,4 \%$ do total das IES, sendo o restante, $12,6 \%$, refere-se às instituições públicas. Quanto a classificação acadêmica, 84,3\% são faculdades, 8,2\% universidades, $5,9 \%$ centros universitários e 1,7\% IF e CEFET. Quanto ao número de ingressos nos cursos de graduação. Houve um aumento no número de ingressos entre os anos de 2010 e 2012 e em 2013, houve estabilidade em relação ao ano anterior, quanto ao número que instituições teve uma redução (CENSO DA EDUCAÇÃO SUPERIOR, 2013).

É relevante pensar nas matrículas de graduação que vêm aumentando nos últimos anos, as matrículas de graduação atingiram o total de 7.305.977 no ano de 2013. De 2010 para 2011, observou-se um crescimento de 5,6\%; de 2011 para 2012, de 4,4\%; e, de 2012 para 2013, 3,8\%, porém, a taxa de crescimento do número de matrículas tem diminuído ano após ano (CENSO DA EDUCAÇÃO SUPERIOR, 2013). Se o número de instituições está diminuindo nos últimos anos e a taxa de matrículas vem caindo, a concorrência nesse mercado vem aumentando.

Nesse contexto é imprescindível que a administração universitária e seus gestores aumentem seus níveis de eficiência e eficácia, resultando na excelência dos serviços prestados. De acordo com a empresa de consultoria Hoper, o Brasil é o país com maior número de instituições de ensino superior com fins lucrativos do mundo (HOPER, 2012). A grandeza dos números que o setor privado de ensino superior exibe hoje deixa claro que o negócio não é para amadores. A profissionalização da gestão se trata de uma obrigação e necessidade.

$$
\text { Para Sampaio (2013), a }
$$
profissionalização da gestão é parte constitutiva e ao mesmo tempo catalizadora das transformações do setor privado hoje; está por trás de toda a movimentação econômica e financeira do setor e é um dos principais fatores desencadeadores das diferenças entre os estabelecimentos. 
Se o mercado de ensino superior hoje exige cada vez mais a gestão profissional dos estabelecimentos. Quanto maior a capacidade das IES se adaptarem aos novos ambientes jurídico-normativo e de mercado, maiores são, evidentemente, as suas chances de sobrevivência e êxito (SAMPAIO, 2013). Nesse sentido, compreender, como se dá a capacidade de adaptação ao ambiente externo em IES, é relevante para pesquisas teóricas e empíricas.

\subsection{Absorção do conhecimento em IES}

As demandas da sociedade brasileira, no que diz respeito ao ensino superior, contribuíram para um ambiente competitivo de faculdades e universidades (NUNES, 2008). O rápido crescimento do investimento particular financiado por grandes grupos educacionais tem provocado alterações profundas no setor. Nesse cenário competitivo, as IES procuram sobreviver no mercado e investem em práticas de gestão (HICKSON, 2014; RIBEIRO, 1999).

Para Castell (2001), a busca pela produtividade, eficiência e eficácia institucional leva as instituições à revisão dos modelos e metodologias com vistas ao planejamento e tomada de decisões, utilizando como principal recurso a informação e do conhecimento (HICKSON, 2014). Considerando que as organizações, que gerenciam seus conhecimentos e conseguem combiná-los e compartilhá-los entre os seus colaboradores, adquirem benefícios, como aumento da competitividade; auxílio na tomada de decisão; melhoria no atendimento das expectativas dos clientes e melhoria da eficiência nas operações (MOTA, 2013). Se faz necessário nesse momento a identificação das práticas de gestão do conhecimento uma sistematização dessas nas IES. Choo (2003) destaca que a eficácia do processo de gestão do conhecimento pode ser melhorada por meio de iniciativas de gerenciamento sistemático.

Contribuindo Mota (2013), ressalta que as dimensões da conversão do conhecimento em IES têm impacto positivo na inovação tecnológica e com o desempenho das IES. Ainda Davenport e Prusak (1998) mencionam também que há uma vantagem sustentável proporcionada pela gestão adequada do conhecimento. Complementam, que a empresa gestora do conhecimento passará para um nível de qualidade, criatividade e eficiência mais elevado que as demais. Que o conhecimento ao contrário dos ativos materiais, que diminuem à medida que são usados, tende a aumentar com a sua utilização e sistematização. 
No contexto apresentado pelos autores, cabe as IES buscarem soluções adequadas para gestão do conhecimento, que proporcionem a aquisição, assimilação, criação e compartilhamento deste, auxiliando no sucesso e desempenho. Portanto, pesquisas que auxiliam na sistematização da gestão do conhecimento para IES são determinantes e necessárias.

\section{PROCEDIMENTOS METODOLÓGICOS}

O estudo é uma revisão da literatura, realizada através de técnicas bibliométricas, com objetivo de mapear e analisar pesquisas já realizadas sobre o tema CA no contexto de IES.

Segundo Kobashi e Santos (2006), por meio da bibliometria, é possível identificar "todos os autores que trabalham em determinado assunto; os periódicos que publicaram este assunto; os autores considerados referência no assunto" e todas as possibilidades de cruzamento entre as variáveis envolvidas. Um estudo bibliométrico é uma forma de mensuração da pesquisa acadêmica, um tema de vital importância e pode ser aplicado a todas as disciplinas (ANDRÉS, 2009). Estudos nesse campo, vem se consolidando como um método quantitativo e estatístico usado para a medição dos índices de produção e disseminação de conhecimentos científicos (ARAÚJO, 2006).

Para isso, utiliza-se de indicadores e dados bibliográficos com o objetivo de traçar a trajetória do desenvolvimento da produção científica, além de realizar análises dos estudos relevantes relacionados aos temas da pesquisa.

Para atingir o objetivo do estudo que é analisar a produção acadêmica sobre capacidade absortiva no contexto das IES, de modo a traçar um mapa dos gaps para novas contribuições acadêmicas na área. Foi utilizado o método de pesquisa bibliométrica, como um termômetro da pesquisa acadêmica no tema capacidade absortiva em IES.

Com procedimentos e técnicas semelhantes as utilizadas em estudos de análise da literatura já realizados sobre o constructo Capacidade Absortiva como Fritsch e Santos (2015), Kurtz; Santos; Steil (2013), Moré et al. (2013), Versiane et al. (2010), Carvalho et al (2015), este trabalho foi realizado em quatro etapas, descritas a seguir.

\section{Etapa 1: Definições iniciais}

Definição do objetivo; Leitura inicial para interação com o tema; Definição das palavras-chaves e definição das bases de dados a serem utilizadas.

Etapa 2: Coleta de dados 
A coleta de dados foi realizada nas datas de 04/02/2016 e 06/02/2016. Os termos de busca utilizados foram Capacidade Absortiva, Instituições de Ensino Superior, Absorptive Capacity e Higher Education Institutions. As bases de dados pesquisadas foram: Spell; Scielo; ScienceDirect; Ebsco; Scopus e Web of Science. Os filtros utilizados durante as buscas foram os seguintes: não foi definida nenhuma data durante a pesquisa, as bases foram filtradas para buscar apenas artigos sobre o tema, além disso, os termos foram pesquisados se estivessem contidos no título do artigo apenas. O resultado da busca foi uma quantidade de 4231 trabalhos.

Etapa 3: Filtragem, Regras de exclusão

\section{A primeira filtragem:}

- Se deu através do download dos artigos, que estavam disponíveis de forma gratuita e completos. Sendo que, nem todos se encaixavam nesse requisito. Após a realização desse processo restaram 909 trabalhos.

\section{A segunda filtragem:}

- Foram excluídos os artigos analisando apenas os títulos conforme a seguinte regra: Nos artigos que sobre capacidade de absorção, foram descartados todos os artigos que não tratavam do tema no ambiente de firmas. Por exemplo: absorção de alimentos, intestino, países, regiões. Além disso, nos trabalhos envolvendo o tema IES, foram descartados os estudos que não se referiam a gestão das instituições. Após essa filtragem restaram 413 trabalhos.

\section{A terceira filtragem:}

- O critério de exclusão utilizado foi a leitura do resumo e se necessário de todo o estudo. Quanto ao tema capacidade absortiva foram descartados estudos que não estavam ligados a nenhuma das áreas da capacidade abortiva como mensuração, conceituação e estudos empíricos realizados sobre o tema. Quanto ao assunto Instituições de ensino superior, foram descartados estudos que não incluíam gestão do conhecimento, aprendizagem organizacional e intangíveis. Restando um total de 26 artigos.

\section{Etapa 4: Sistematização dos} estudos

Após a tabulação dos estudos em uma planilha foram identificados os locais das publicações, os periódicos, o ano de publicação, os métodos utilizados, assim como as referências de todos artigos. Com isso foram geradas as tabelas que são apresentadas na análise de resultados.

Quanto a seleção das bases de dados, foram selecionadas por serem as bases que retornaram mais resultados durante uma busca prévia sobre os assuntos. A seleção dos termos de busca foi definida 
com base nas pesquisas já realizadas utilizando o mesmo método, porém quando utilizadas as palavras chave "Capacidade Absortiva" e "Instituições de ensino superior", em conjunto na mesma busca, os resultados não eram satisfatórios. Sendo assim, antes da realização da busca definitiva, tentativas utilizando os termos de forma isolada foram realizadas, obtendo-se uma gama maior de resultados. Como as bases de dados incluem publicações nacionais e internacionais os termos foram pesquisados no idioma inglês além de português sendo "Absorptive Capacity" $\mathrm{e}$ "Higher Education Institutions", proporcionando resultados mais abrangentes.

\section{ANÁLISE DOS RESULTADOS}

Nesta seção, apresentam-se os resultados e sínteses das informações selecionadas. A Tabela 2 apresenta a quantidade de artigos por base de dados, resultante da etapa 1 da busca. A quantidade inicial encontrada totalizou 4231 artigos. Nesse momento observa-se que as bases Scopus e Web of Science, apresentam um número significativo de publicações. No entanto, no momento da aplicação da primeira filtragem que foi o download de publicações disponíveis de forma livre, apenas 3,62\% dos artigos são considerados, o que de certa forma contribui e pode comprometer resultados de pesquisas sem apoio financeiro ou realizada por pesquisadores que não possuem acesso a essas bases.

Tabela 1 - Quantidade de artigos por base de dados encontrados na pesquisa bibliométrica

\begin{tabular}{lcccc}
\hline Base de dados & Total de itens & $\mathbf{1}^{\mathbf{a}}$ Filtragem & $\mathbf{2}^{\mathbf{a}}$ Filtragem & $\mathbf{3}^{\mathbf{a}}$ Filtragem \\
\hline Spell & 181 & 181 & 87 & 5 \\
Scielo & 195 & 195 & 48 & 2 \\
ScienceDirect & 105 & 104 & 29 & 1 \\
Ebsco & 304 & 304 & 192 & 16 \\
Scopus & 1735 & 100 & 50 & 2 \\
Web of Science & 1711 & 25 & 7 & 0 \\
\hline TOTAL & 4231 & 909 & 413 & 26 \\
\hline
\end{tabular}

Fonte: dados da pesquisa.

Na Tabela 3, foram caracterizados os artigos após a aplicação das três filtragens. Nesse momento, o dado relevante foi o grande número de diferentes periódicos, que de um total de 26 publicações foram encontrados 25 periódicos diferentes, ou seja, apenas dois artigos foram publicados no periódico Academy of Management Review, as demais publicações apenas um por periódico (Tabela 5). 
Tabela 2 - Caracterização dos artigos selecionadas após a filtragem aplicada na pesquisa bibliométrica

\begin{tabular}{|c|c|}
\hline Dados bibliográficos & Frequência \\
\hline Artigos & 26 \\
\hline Locais & 9 \\
\hline Número de períodos & 25 \\
\hline Referências citadas capacidade absortiva & 751 \\
\hline Referências citadas instituições de ensino superior & 193 \\
\hline
\end{tabular}

Fonte: dados da pesquisa.

Quanto ao local de publicação na Tabela 4, pode-se verificar que os EUA possuem $55 \%$ das publicações sobre o tema Capacidade Absortiva em segundo lugar com está o Brasil com 18\%. No entanto no tema Instituições de ensino superior o Brasil se encontra em primeiro lugar com $60 \%$ do número de publicações e os USA com $20 \%$ apenas. No entanto, dos três artigos nacionais sobre capacidade absortiva, dois deles foram realizados de modo empírico em instituições de ensino, que nesse caso são os estudos mais relevantes dessa pesquisa bibliométrica, pois estão relacionados aos dois temas em questão.

O estudo recente realizado por Florindo Rhaoni Picoli e Adriana Takahashi publicado em fevereiro de 2016, teve como objetivo buscar compreender de que maneira se dá o processo recursivo de capacidade de absorção e de aprendizagem organizacional, por meio de mecanismos de integração social, na utilização do Programa Paraná Digital em duas instituições de ensino público de Curitiba - Paraná, no período de 2007 a 2013. O estudo teve como principais correntes a aprendizagem organizacional e a integração social.

Utilizando-se dos autores Lane e Lubatkin (1998), que detectaram uma relação existente entre a capacidade de absorção e a aprendizagem organizacional, de recursividade, ou seja, à medida que uma organização desenvolve seu aprendizado, ela aumenta sua capacidade de compreender e obter novos conhecimentos. Também quanto a integração social os autores (COHEN e LEVINTHAL, 1990 e ZAHRA e GEORGE, 2002) inferiram que a capacidade de absorção potencial só se torna realizada quando é mediada por mecanismos de integração social, que se constituem como elo que possibilita que a empresa assimile, transforme e explore o conhecimento. O estudo comprovou que os mecanismos de integração social desempenham papel essencial para 
viabilizar a aprendizagem e, consequente, $o$ aumento da capacidade de absorção.

Sabrina Rossi de Oliveira e Alsones Balestrin em 2015, publicaram um estudo incluindo a Universidade do Vale do Rio dos Sinos - UNISINOS e um projeto UNISINOS - HT Micron. Com o objetivo de investigar o desenvolvimento da capacidade absortiva de uma universidade brasileira, diante do desafio de atuar em um projeto colaborativo junto à indústria de semicondutores. Nesse estudo a principal corrente da literatura foi que a capacidade absortiva é uma habilidade dependente da trajetória da organização, o que a torna difícil de ser desenvolvida em um espaço relativamente curto de tempo. Os resultados mostraram que a formação da capacidade absortiva não é necessariamente dependente da trajetória da organização, podendo ser desenvolvida, ao menos em parte, no contexto de um empreendimento específico.

Tabela 4 - Relação entre termos de busca e país da publicação

\begin{tabular}{|c|c|c|c|c|c|c|c|c|c|c|c|}
\hline Termo de busca & Holanda & EUA & Canadá & Ásia & Índia & Indonésia & Brasil & Colômbia & Cuba & $\begin{array}{c}\text { TOTA } \\
\text { L }\end{array}$ & $\%$ \\
\hline $\begin{array}{l}\text { Capacidade } \\
\text { Absortiva }\end{array}$ & & & & & & & & & & 0 & $0 \%$ \\
\hline Absorptive Capacity & 1 & 10 & 1 & 1 & & 1 & 3 & 1 & & 18 & $69 \%$ \\
\hline $\begin{array}{l}\text { Higher Education } \\
\text { Institutions }\end{array}$ & & 1 & & & 1 & & 3 & & & 5 & $19 \%$ \\
\hline $\begin{array}{l}\text { Instituições de ensino } \\
\text { superior }\end{array}$ & & & & & & & 2 & & 1 & 3 & $12 \%$ \\
\hline TOTAL & 1 & 11 & 1 & 1 & 1 & 1 & 8 & 1 & 1 & 26 & $\begin{array}{c}100 \\
\%\end{array}$ \\
\hline$\%$ & 3,85 & 38,46 & 3,85 & $\mathbf{3 , 8 5}$ & $\mathbf{3 , 8 5}$ & $\mathbf{3 , 8 5}$ & 34,62 & 3,85 & $\mathbf{3 , 8 5}$ & 100 & \\
\hline
\end{tabular}

Fonte: dados da pesquisa.

Do total $69 \%$ das publicações encontradas foram utilizando o termo de busca Absorptive Capacity, e 19\% com o termo Higher Education Institutions, ou seja, utilizando-se palavras no idioma inglês a busca se torna mais abrangente sobre os temas nas bases pesquisadas, pois somente $12 \%$ dos artigos encontrados foram localizados utilizando os termos em português (Tabela 4).

Os artigos são originários de 25 periódicos diferentes, $28 \%$ deles são brasileiros e $72 \%$ internacionais, apenas um periódico possui duas publicações. Os resultados são apresentados na Tabela 5. 
$\underline{\text { Tabela } 5 \text { - Publicações por periódico }}$

\begin{tabular}{|c|c|}
\hline Nome do periódico & Número de publicações \\
\hline Academy of Management Journal & 1 \\
\hline Academy of Management Review & 2 \\
\hline Administrative Science Quarterly & 1 \\
\hline Elsevier & 1 \\
\hline European Management Journal & 1 \\
\hline Faculty of Social and Political Sciences & 1 \\
\hline Gest. Prod., São Carlos & 1 \\
\hline Global Institute of Information Technology & 1 \\
\hline Intellect Limited & 1 \\
\hline International Journal of Innovation Management & 1 \\
\hline Journal of Managerial Issues & 1 \\
\hline Management international & 1 \\
\hline Organization Science & 1 \\
\hline Perspective Organization Science & 1 \\
\hline$R \& D$ Management & 1 \\
\hline RAC & 1 \\
\hline RAI - Revista de Administração e Inovação & 1 \\
\hline RAM, Rev. Adm. Mackenzie & 1 \\
\hline Rev. Cent. Ciênc. Admin & 1 \\
\hline Revista Científica de la Universidad de Cienfuegos & 1 \\
\hline Revista de Administração de Empresas & 1 \\
\hline Revista de negócios - FURB & 1 \\
\hline $\begin{array}{l}\text { Revista Eletrônica de Ciência Administrativa } \\
\text { (RECADM) }\end{array}$ & 1 \\
\hline $\begin{array}{l}\text { Revista Globalização, competitividade e } \\
\text { governabilidade }\end{array}$ & 1 \\
\hline SAM Advanced Management Journal & 1 \\
\hline
\end{tabular}

Fonte: dados da pesquisa.

Quanto ao período das publicações os dados da Tabela 6 mostram que os temas estão sendo pesquisados recentemente, apesar de o artigo seminal ter sido publicado em 1990 por Cohen e Levinthal no periódico Administrative Science Quarterly dos EUA. Pode-se verificar que a partir de
2011 as publicações envolvendo Capacidade Absortiva e Instituições de Ensino começaram a ter um aumento significativo e no ano de 2015 a porcentagem de publicação que é de $30,77 \%$, do total encontrado. 
Tabela 6 - Período das publicações

\begin{tabular}{ccc}
\hline Ano & Número de publicações & \% \\
\hline 2015 & 8 & 30,77 \\
2014 & 2 & 7,69 \\
2013 & 3 & 11,54 \\
2012 & 1 & 3,85 \\
2011 & 2 & 7,69 \\
2010 & 1 & 3,85 \\
2008 & 1 & 3,85 \\
2007 & 1 & 3,85 \\
2006 & 1 & 3,85 \\
2005 & 1 & 3,85 \\
2004 & 1 & 3,85 \\
2003 & 1 & 3,85 \\
2002 & 1 & 3,85 \\
1999 & 1 & 3,85 \\
1990 & 1 & 3,85 \\
\hline
\end{tabular}

Fonte: dados da pesquisa.

A partir da leitura e análise dos 26 artigos selecionados no estudo foi possível identificar que somente $4 \%$, ou seja, um dos artigos utilizou o método bibliométrico. Já estudos quantitativos com uso de survey totalizou 58\%, com aplicação de questionários em empresas localizadas em diferentes países. O método de revisão bibliográfica foi utilizado em $23 \%$ e o método de estudo de caso em $15 \%$ dos artigos (Tabela 7).

Autores como Lane et al. (2001), Van Den Bosch, Volberda e De Boer (1999), relatam que diferentes dimensões da capacidade absortiva não foram ainda testadas empiricamente. Observa-se na pesquisa que os métodos Survey, que tratam da aplicação de questionários, ou seja, estudos empíricos estão sendo realizados com mais frequência nos últimos anos. A validação das dimensões e componentes do constructo de capacidade absortiva, no ano de 2013 as publicações forma de 11,54\% e no ano de 2015 aumentaram para 30,77\%.

$\mathrm{O}$ que vem de acordo com Chauvet (2014), pois o autor menciona que várias pesquisas e modelos de mensuração do constructo capacidade absortiva foram desenvolvidos, agora é hora de validá-los através de pesquisas empíricas. Além disso, pode-se observar que as pesquisas de estudo de caso e survey se encontram nas pesquisas mais recentes, no caso de pesquisas survey oito artigos foram publicados nos últimos cinco anos equivalendo a $30,77 \%$ dos estudos encontrados (Tabela 7).

Dois artigos utilizaram a revisão da literatura, abrangendo o tema instituições de ensino. Já no constructo capacidade absortiva quatro artigos fazem uma revisão da literatura com ênfase na definição de um framework para mensuração. Os estudos de caso foram todos realizados sobre o tema 
capacidade absortiva, conforme os dados da

Tabela 7.

Tabela 7 - Métodos utilizados nos artigos encontrados na pesquisa bibliométrica

\begin{tabular}{lccc}
\hline \multicolumn{1}{c}{ Método } & $\mathbf{N}^{\mathbf{0}}$ de publicações & \% & Período \\
\hline Bibliometria & 1 & 3 & 2010 \\
Estudo de caso & 4 & 15,4 & $1999-2014-2015$ \\
Revisão da literatura com proposta de & 4 & 15,4 & $2002-2006-2007-2015$ \\
modelos & 2 & 7,7 & 1990 e 2015 \\
Conceituação e definição & 15 & 57,7 & $2003-2004-2005-2008-2011-2012-$ \\
Survey & & & 2015 \\
\hline
\end{tabular}

Fonte: dados da pesquisa.

No quadro 2, são mostradas as publicações mais citadas nos artigos referentes ao tema capacidade absortiva analisados neste estudo. As primeiras quatro obras possuem destaque no campo. Outros autores até possuem destaque, em diferentes obras, como exemplo de Van Den Bosh, Lane e Volberda. Além dos autores mostrados na tabela existem os que foram utilizados como referência citados apenas duas vezes com publicações diferentes como exemplo de Winter S. (2000, 2003), Matusik (1998,2005), Lyle (1996,1992).

Quadro 2 - Autores mais citados no tema capacidade absortiva nos artigos encontrados na pesquisa bibliométrica

\begin{tabular}{|l|c|}
\hline \multicolumn{1}{|c|}{ Autores } & $\mathbf{N}^{\circ}$ de artigos \\
\hline Cohen W H and Levinthal D A (1990) & 16 \\
\hline Zahra S A and George G (2002), & 13 \\
\hline Grant, R. (1996). & 11 \\
\hline Lane, P. J., B. R. Koka, and S. Pathak (2006) & 9 \\
\hline $\begin{array}{l}\text { Jansen J. J. P., Van Den Bosh F. A. J. and Volberda H W (2005); Lane, P. J., \& Lubatkin, M. } \\
\text { (1998) }\end{array}$ & 8 \\
\hline $\begin{array}{l}\text { Cohen, M. W., \& Levinthal, D. A. (1989); Van den Bosch, F. A. J., Volberda, H. W., \& Boer, } \\
\text { M. de (1999) }\end{array}$ & 7 \\
\hline $\begin{array}{l}\text { Lane P J, Salk J E and Lyles M A (2001); Nonaka I (1994); Szulanski, G. (1996); Tsai, W } \\
\text { (2001). }\end{array}$ & 6 \\
\hline $\begin{array}{l}\text { Camisón, C. and Forés, B. (2010); Eisenhardt, K. M. and J. A. Martin. (2000); Flatten, T. C., } \\
\text { A. Engelen, S. A. Zahra, and M. Brettel. (2011); Mowery, D. C, \& Oxley, J. E. (1995); Spender, } \\
\text { J. C. (1996); Todorova, G. and B. Durisin. (2007) }\end{array}$ \\
\hline $\begin{array}{l}\text { Dyer J., and Singh, H. (1998); Jiménez-Barrionuevo, M. M., García-Morales, V. J. and Molina, } \\
\text { L. M. (2011); Liao, J., H. Welsch, and M. Stoica (2003); Liao, S., Fei, W., \& Chen, C. (2007); } \\
\text { Lichtenthaler, U (2009); Teece, D. J., Pisano, G., \& Shuen, A. (1997); Veugelers, R. (1997) }\end{array}$ \\
\hline
\end{tabular}


(CONTINUAÇÃO)

Autio, E., H. J. Sapienza, and J. G. Almeida. (2000); Hansen, M. T. (1999); Kogut, B. and U Zander (1992); March, H. G. (1991); Mowery, D. C, Oxley, J. E. \& Silverman. B. S. (1996); Nelson, R. R., S. G. Winter (1982); Nunnally J. (1978); Podsakoff, P. M., S. B. MacKenzie, J. Y. Lee, and N. P. Podsakoff (2003); Stock, G., Greis, N.P. and Fischer, W.A. (2001); Volberda, H. W., N. J. Foss, and M. A. Lyles (2010)

Argote, L, B McEvily and R Reagans (2003); Armstrong, J., \& Overton, T. (1977); Barney, J. (1991); Boynton, A., Zmud, R., \& Jacobs, G. (1994); Churchill, G. (1979);

Cockburn, I., Henderson, R. and Stern, S. (2000); Cohen, W. M., D. A. Levinthal (1994);

Fosfuri, A. and J. A. Tiibó (2008); George, G., Zahra, S. A., Wheathley, K. K., \& Khan, R. (2001); Gupta, A. K., \& Govindarajan, V. (2000); Henderson, R. M., \& Clark, K. B. (1990); Jaworski, B. J., \& Kohli, A. K. (1993); Khandwalla, P. N. (1977); Kim, L. (1998); Lenox, M. and A King (2004); Mangematin, V. and Nesta, L. (1999); Minbaeva D., Pedersen T., Bjorkman I, Fey C F and Park H J (2003); Murovec, N. and Prodan, I. (2009); Nahapiet, J. and S. Ghoshal (1998); Schmidt, T. (2005); Van Den Bosch, F. A. J., Van Wijk, R., \& Volberda, H. W. (2003); Vega-Jurado, J., Gutiérrez-Gracia, A., \& Fernández-de-Lucio, I. (2008); Wang, C. L., \& Ahmed, P. K. (2007);

Zander, U. and Kogut, B. (1995); Zollo, M. and Winter, S. G. (2002).

Fonte: dados da pesquisa.

Autores mais citados no tema Instituições de Ensino Superior foram Villafañe (5 obras); Spender (3 obras); Sanchez (4 obras); Nonaka (6 obras); Davenport (3 obras); Cunha (6 obras); Cañibano (5 obras). Não necessitando elaboração de uma tabela para apresentação, porque cada obra foi citada apenas uma vez, ou seja, o mesmo autor até foi citado no mesmo artigo, porém em obras diferentes. No item a seguir são apresentadas as considerações, limitações e sugestões para estudos futuros abrangendo os dois campos de pesquisa.

\section{CONSIDERAÇÕES FINAIS}

Os estudos bibliométricos vêm se consolidando como um método quantitativo usado para medir índices de produção e disseminação de conhecimentos científicos (ARAÚJO, 2006). O objetivo do estudo é analisar a produção acadêmica sobre capacidade absortiva no contexto das IES, de modo a traçar um mapa dos gaps para novas contribuições acadêmicas na área.

Foi possível identificar as obras mais citadas sobre os temas, após a sistematização dos autores. Dado importante para localização dos principais trabalhos, inclusive no tema capacidade absortiva. Essa informação é relevante para quem procura se aprofundar no assunto, pois se trata da identificação de autores que estudam apenas esse tema e são especialistas.

Quanto às publicações localizadas, nota-se que são recentes, tendo um aumento significativo a partir do ano de 2011. Principalmente em obras utilizando métodos empíricos para validar modelos de mensuração, esse fato mostra que a 
mensuração da capacidade absortiva é recente e, no ambiente de instituições de ensino, mais recente ainda, pois os estudos localizados são de 2015 e 2016.

Isso corrobora o que Chauvet (2014) propôs. Para ele, várias pesquisas e modelos de mensuração do constructo capacidade absortiva foram desenvolvidas. Por exemplo, Van Den Bosch; Volberda; De Boer (1999) desenvolveram um modelo mais integrado da capacidade de absorção dependente da trajetória de uma empresa e do ambiente do conhecimento. Hopkins e Gross (2015), por sua vez, sugerem aplicar estudos empíricos envolvendo o constructo em diferentes contextos, inclusive propondo um framework com essa finalidade. Vega-Jurado; Gutiérrez-Gracia; Fernándes-de-Lucio (2008) propõem um modelo para analisar os determinantes da capacidade absortiva. E, conforme Chauvet (2014), agora é hora de validá-los em diferentes contextos, pois modelos e frameworks já existem.

$\mathrm{O}$ método utilizado tem seu ponto fraco no acesso às bases de dados. A primeira filtragem exclui um número significativo de estudos quando são selecionados apenas aqueles disponíveis para download de forma completa e gratuita. Para a realização de uma pesquisa mais robusta, deve utilizar-se um usuário com acesso total às bases de dados. Quanto à localização dos artigos contendo os termos no seu título, alguns trabalhos podem não ter sidos listados durante a busca, se o título não continha o termo.

O resultado relevante da pesquisa está no fato de dois estudos apenas serem encontrados envolvendo o constructo capacidade absortiva em instituições de ensino superior, com as regras impostas durante a pesquisa. Ambos foram realizados de forma empírica, envolvendo instituições de ensino, no setor acadêmico das instituições, ou seja, não envolveram a instituição como um todo.

$\mathrm{O}$ estudo destaca o fato de nem sempre a capacidade absortiva depender do caminho percorrido pela organização, conforme propõem Cohen e Levinthal (1990). Os resultados mostraram que a capacidade absortiva foi aperfeiçoada por meio da capacitação de recursos humanos, podendo ser desenvolvida, ao menos em parte, no contexto de um conhecimento específico (OLIVEIRA; BALESTRIN, 2015). Já no estudo realizado por Picoli e Takahashi (2016), verificou-se a complementaridade do processo de capacidade de absorção mencionada por Zhara e George (2002), em que as falhas em um processo impactam o outro, demonstrando que é mediada por mecanismos de integração social (FILENGA, 2009). 
As IES necessitam melhorar seus processos de gestão (RIBEIRO, 1999). A busca pela produtividade, eficiência e eficácia pode ser auxiliada pela utilização da informação e do conhecimento (CASTELL, 2001; HICKSON, 2014). Isso porque as organizações, que gerenciam seus conhecimentos, adquirem benefícios como: aumento da competitividade, auxílio na tomada de decisão, melhoria no atendimento das expectativas dos clientes e melhoria da eficiência nas operações (MOTA, 2013). Para Davenport e Prusak (1998), há uma vantagem sustentável proporcionada pela gestão adequada do conhecimento. Cabe às IES buscar soluções adequadas para gestão desse recurso intangível.
As IES serão cada vez mais instáveis, e os seus membros cada vez mais forçados a desviar energias das tarefas intelectuais e sociais da universidade para as tarefas organizativas e institucionais. Diante disso, estudos podem contribuir para que as tarefas organizativas se realizem com o objetivo de prover às instituições competitividade e inovação (SANTOS, 1999).

Nesse sentido, estudos futuros para identificar práticas de absorção e gestão do conhecimento nas IES são de extrema relevância para suprir a lacuna teórica existente. O mesmo vale para pesquisas empíricas que busquem validar modelos propostos para identificação e mensuração das práticas de CA (CHAUVET, 2014). 


\section{REFERÊNCIAS}

AGUIAR FILHO, Armando Sérgio de et al. Práticas de gestão da informação e do conhecimento nos processos de avaliações do INEP/MEC: um estudo em uma instituição de ensino superior. XIV COLÓQUIO INTERNACIONAL DE GESTÃO UNIVERSITÁRIA - CIGU, 2014.

AHUJA, G.; LAMPERT, C.M. Entrepreneurship in the large corporation: A longitudinal study of how established firms create breakthrough inventions. Strategic Management Journal, 22, p. 521-543. 2001.

ANDRÉS, A. Measuring academic research: How to undertake a bibliometric study. Elsevier. 2009.

ARAÚJO, C.A. Bibliometria: evolução histórica e questões atuais. Em Questão, 12(1). 2006.

BARRETO, Adriano Cardoso. Gestão do conhecimento mapeamento do conhecimento em uma instituição de ensino superior da região centro-oeste. Universidade Fumec Faculdade de Ciências Empresariais. Mestrado profissional em sistemas de informação e gestão do conhecimento Mapeamento. Belo Horizonte-MG, 2015.

BERTOLIN, J.C.G.. A Avaliação da Qualidade do Sistema de Educação Superior Brasileiro em Tempos de Mercantilização - Período 1994-2003. Tese de Doutorado. Universidade Federal do Rio Grande do Sul Faculdade de Educação. Programa de PósGraduação em Educação. 2007, 282 p.

CAMPION, M. A.; CHERASKIN, L.; STEVENS, M. J. Career-related antecedents and outcomes of job rotation. Academy of Management Journal, 37, p. 1518-1542. 1994.

CARDOSO, Olinda Nogueira Paes; MACHADO, Rosa Teresa Moreira. Gestão do conhecimento usando data mining: estudo de caso na Universidade Federal de Lavras. Revista de Administração Pública, 42(3), p. 495-528, 2008.

CENSO DA EDUCAÇÃO SUPERIOR. . 2013 - resumo técnico. Brasília: Instituto Nacional de Estudos e Pesquisas Educacionais Anísio Teixeira, 2013. Disponível em: $<$ http://download.inep.gov.br/download/superior/censo/2013/resumo _tecnico_censo_educacao_superior_2013.pdf> Acessado em: 31 mar. 2016. 
CENSO DA EDUCAÇÃO SUPERIOR. 2007 - Resumo técnico. Brasília: Instituto Nacional de Estudos e Pesquisas Educacionais Anísio Teixeira, 2013. Disponível em: <http://download.inep.gov.br/download/superior/censo/2007/Resumo_tecnico_2007.pdf>. Acessado em: 31 mar. 2016.

CENSO DA EDUCAÇÃO SUPERIOR. 2004 - Resumo técnico. Brasília: Instituto Nacional de Estudos e Pesquisas Educacionais Anísio Teixeira, 2013. Disponível em: $<$ http://download.inep.gov.br/download/superior/2004/censosuperior/Resumo_tecnicoCenso_2004.pdf >. Acessado em: 31 mar. 2016.

CHAUVET, V. Absorptive Capacity: Scale Development and Implications for Future Research. Capacidad de Absorción: Propuesta de Medida Y Contribuciones a Futuras Investigaciones, 19(1), p. 113-129, 2014.

CHOO, C. W. A Organização do conhecimento: como as organizações usam a informação para criar significado, construir conhecimento e tomar decisões. Editora Senac, São Paulo, 2003.

COHEN, M.D.; BACDAYAN, P. Organizational routines are stored as procedural memory. Organization Science, 5, p. 554-568. 1994.

COHEN, W.; LEVINTHAL, D. Absorptive Capacity: A New Perspective on Learning and Innovation. Science, 35(1), p. 128-152. 1990.

CUNHA, Luiz Antônio. Ensino Superior e a Universidade no Brasil. In: LOPES, E. M. T. et al. 500 Anos de Educação no Brasil. Belo Horizonte: Autentica, 2000.

DAVENPORT, T. H.; PRUSAK, L. Conhecimento empresarial. Rio de Janeiro: Campus, 1998.

OLIVEIRA, Sabrina Rossi de; BALESTRIN, Alsones. Cooperação universidade-empresa: um estudo do projeto UNISINOS - HT Micron para o desenvolvimento de capacidade absortiva na área de semicondutores. Gestão \& Produção, São Carlos, 2015.

DOMINGUES, M.J.C.S; MONDINI, V.E.D. Entendendo a classificação das IES no brasil. Poder, Gobierno Y Estrategias En Las Univeridades de America Del Sur, 1(September 2013), p. 1-36. 2013.

ETZIONI, Amitai. Organizações modernas. São Paulo: Pioneira, 1989. 
FILENGA, D. Capacidade absortiva, mecanismos de integração social e desempenho inovativo empresarial brasileiro, 2009. Disponível em: <http://www.altec2013.org/programme_pdf/822.pdf>. Acessado em: 20 abr. 2016.

HOPER. Análise setorial do ensino superior privado. Brasil. 2012.

JANSEN, J. J. P.; VAN DEN BOSCH, F. A. J.; VOLBERDA, H. W. Managing potential and realized absorptive capacity: How do organizational antecedents matter? Academy of Management Journal, 48, p. 999-1015. 2005.

KOBASHI, Nair Yumiko e SANTOS, Raimundo Nonato Macedo dos. Institucionalização da pesquisa científica no Brasil: cartografia temática e de redes sociais por meio de técnicas bibliométricas. Transformação [online]. v. 18, n. 1, p. 27-36. 2006.

KOZA, M.; LEWIN, A. Y. The coevolution of strategic alliances. Organization Science, 9, p. 255-264. 1998.

LANE, P. J.; KOKA, B. R.; PATHAK, S. The reification of absorptive capacity: a critical review and rejuvenation of the construct. Academy of Management Review, v. 31, n. 4, p. 833-863, 2006.

LANE, P.; LUBATKIN, M. Relative absorptive capacity and inter-organizational learning. Strategic Management Journal, 19 (5), p. 111-125. 1998.

LEMAITRE, Maria J. La Calidad Colonizada: universidad y globalizacion. Conferencia dictada en el Seminario. The End of Quality, organizado por la Universidad de Central England, Birmingham, U.K. en mayo de 2001.

LICHTENTHALER, Ulrich; LICHTENTHALER, Eckhard. A capability-based framework for open innovation: complementing absorptive capacity. Journal of Management Studies, v. 46, n. 8, Dec. 2009.

MOTTA, M.E.V. da. Capacidade de conversão do conhecimento, inovação tecnológica e o desempenho das IES do sul do Brasil. (Tese de doutorado). Universidade de Caxias do Sul. Rio Grande do Sul, 2013. 
MUROVEC, N.; PRODAN, I. Absorptive capacity, its determinants, and influence on innovation output: cross-cultural validation of the structural model, Technovation, 29 (12), p. 859-872. 2009.

NONAKA, I.; TAKEUCHI, H. Criação de conhecimento nas empresas: como as empresas japonesas geram a dinâmica da inovação. Rio de Janeiro: Campus, 1997. 358 p.

NUNES, G. T. Abordagem do marketing de relacionamento no ensino superior. Gestão e Regionalidade, v. 24, n. 69, jan./abr. 2008.

NUNES, S. (2012). A capacidade de absorção e as orientações associadas à exploração (exploration) e à explotação (exploitation) do conhecimento organizacional: uma análise em empresas de Palmas/TO. Tese de doutorado - Universidade Mackenzie, Programa de pósgraduação em Administração, 2012.

PENNINGS, J.M.; HARIANTO, F. Technological networking and innovation implementation. Organ. Sci., 3, p. 356-382. 1992.

RIBEIRO, M. Universidade brasileira pós-moderna: democratização $\times$ competência. Manaus: Universidade do Amazonas, 1999.

RODRIGUES, L.C.; MACCARI, E.A. Gestão do Conhecimento em Instituições de Ensino Superior. Revista de Negócios, 8 (2). 2003.

SAMPAIO, H. O setor privado de ensino superior no Brasil: Continuidades e transformações. Revista Ensino Superior Unicamp, 1, p. 28-43. 2011.

SAMPAIO, H. Setor privado de ensino superior no Brasil: crescimento, mercado e Estado entre dois séculos. Ensino Superior: Expansão, Diversificação, .., 7, p. 1-26. 2013. Disponível em: $<$ http://nupps.usp.br/downloads/relatorio2013/Anexo_44_Texto para Livro Maria Ligia Barbosa.pdf> Acessado em: 18/03/2016.

SCHWARTZMAN, S. Policies for Higher Education in Latin America: the Context. Higher Education, 25, p. 9-20, 1993.

SZULANSKI, G. Exploring internal stickiness: Impediments to the transfer of best practice within the firm. Strategic Management Journal, 17, p. 27-43. 1996. 
TOBÍO, Alfonso G.; PÉREZ, Juan C. P. (Org.). As políticas neoliberais na Universidade. Disponível em: <http://firgoa.usc.es/drupal/node/2444>. Acesso em: 14 abr. 2016.

TSENG, S.M. The correlation between organizational culture and knowledge conversion on corporate performance. Journal of Knowledge Management, Kempston, v. 14, n. 2, p. 269$284,2010$.

VAN DEN BOSCH, F.; VOLBERDA, H.; DE BOER, M. Co-evolution of firm absorptive capacity and knowledge environment: organizational forms and combinative capabilities. Organization Science, 10 (5), p. 551-568. 1999.

VEGA-JURADO, Jaider; GUTIÉRREZ-GRACIA, Antonio; FERNÁNDES-de-LUCIO, Ignácio. Analyzing the determinants of firm's absorptive capacity: beyond R\&D. R\&D Management, v. 38, n. 4, p. 392-405, 2008.

WEICK, K. E. The social psychology of organizing. Reading, MA: Addison-Wesley, 1979.

WORMELL, I. Informetria: explorando bases de dados como instrumentos de análise. Ciência da Informação, v. 27, n. 2, p. 210-216. 1998.

ZABALBA, Miguel A. O ensino universitário: seu cenário e seus protagonistas. Porto Alegre: Artmed, 2004.

ZAHRA, S. A.; GEORGE, G. Absorptive capacity: A review, reconceptualization, and extension. Academy of Management Review, 27 (2), p. 185-203. 2002. 